\title{
Validación para la utilización en Colombia de la escala EORTC QLQ-ST022 para la evaluación de la calidad de vida de pacientes con cáncer de estómago
}

\author{
Colombian Ualidation of the EORTC QLQ-STO22 for Eualuating Quality \\ of Life in Patients Diagnosed with Gastric Cancer \\ Validação para a utilização na Colômbia da escala EORTC QLQ-STO22 para \\ a avaliação da qualidade de vida de pacientes com câncer de estômago
}

\author{
Claudia Ibáñez* \\ Instituto Nacional de Cancerología \\ Ricardo Sánchez \\ Universidad Nacional de Colombia \\ Ricardo Oliveros* \\ Instituto Nacional de Cancerología
}

Doi: dx.doi.org/10.12804/apl33.03.2015.03

\section{Resumen}

Objetivo: Validar la escala EORTC QLQ-STO22 para medir la calidad de vida en pacientes con cáncer de estómago en Colombia. Métodos: A 442 pacientes con diagnóstico de cáncer de estómago (entre 26 y 90 años), les fue aplicado el cuestionario EORTC QLQ-STO22. Se evaluó la validez de contenido con técnicas de análisis factorial; la validez de criterio concurrente comparando puntuaciones de la escalas QLQ-STO22 y FACIT-Ga; la consistencia interna mediante alfa de Cronbach, la confiabilidad prueba-reprueba por medio del coeficiente de correlación concordancia de Lin y la sensibilidad al cambio luego de una intervención terapéutica comparando medias en mediciones repetidas. Resultados: El análisis factorial confirmó una estructura de cinco dimensiones y el análisis confirmatorio mostró aceptable ajuste del modelo. Las correlaciones más fuertes entre la escala EORTC QLQ-STO22 y FACIT-Ga se observaron con los dominios "Estado físico general de salud" y la subescala específica para cáncer gástrico de la escala FACIT-Ga. Se encontró alta consistencia interna con valores de alfa de Cronbach de la escala global de .897. La medición de la confiabilidad prueba-reprueba reportó valores del coeficiente de correlación concordancia de Lin entre .656 y .851. Las diferencias entre puntuaciones antes y después de una intervención terapéutica solo fueron significativas para los dominios disfagia y restricciones para comer. Conclusión: La escala EORTC QLQ-STO22 es un instrumento válido y confiable para medir calidad

* Claudia Ibáñez, miembro del Grupo de Investigación Clínica, Instituto Nacional de Cancerología, Colombia; Ricardo Sánchez, miembro del Grupo de Investigación Clínica, Instituto Nacional de Cancerología, Colombia, y Universidad Nacional de Colombia; Ricardo Oliveros, miembro del Grupo de Cirugía Gastrointestinal y Endoscopia Digestiva, Instituto Nacional de Cancerología.

La correspondencia relacionada con este artículo debe ser enviada a Ricardo Sánchez. Correo electrónico: rsanchezpe@unal.edu.co

Cómo citar este artículo: Ibáñez, C., Sánchez, R. \& Oliveros, R (2015). Validación para la utilización en Colombia de la escala EORTC QLQ-STO22 para la evaluación de la calidad de vida de pacientes con cáncer de estómago. Avances en Psicología latinoamericana, 33(3), 397-411. doi: dx.doi.org/10.12804/ap133.03.2015.03 
de vida en pacientes colombianos. Se recomienda efectuar estudios adicionales para confirmar los hallazgos reportados durante la evaluación de la sensibilidad al cambio de esta escala en población colombiana.

Palabras clave: calidad de vida; cáncer gástrico; validación de escalas; cuestionario; EORTC QLQ-STO22.

\section{fibstract}

Objective: To validate the EORTC QLQ-STO22 for measuring quality of life in patients with gastric cancer in Colombia. Method: Factor analysis (exploratory and confirmatory) was used to evaluate content validity. Concurrent validity was evaluated measuring the correlation between QLQ-STO22 and FACIT-Ga. Cronbach's alpha was used to evaluate internal consistency. Test-retest reliability was measured using the Lin concordance-correlation coefficient. Sensitivity to change was evaluated comparing the medians pre-and post-treatment. Results: The QLQ-STO22 was responded by 442 patients with diagnostic of gastric cancer. Exploratory factor analysis revealed five domains and confirmatory factor analysis indicated an acceptable fit to the data. A high internal consistency with Cronbach's alpha of .897was found. Stronger correlations were found between QLQ-STO22 (general health status) and FACIT-Ga (specific subscale of gastric symptoms and related problems). For test-retest reliability Lin coefficients between .656 and .851 were found. Differences between pre-and post-therapy scores were significant for some of the domains of the scale (dysphagia, restrictions on feeding). Conclusion: The EORTC QLQ-STO22 is a reliable and valid instrument for measuring quality of life in Colombian patients. Further studies aimed at evaluating sensitivity to change are recommended. Key words: Quality of life; gastric cancer; scale validation; questionnaire; EORTC QLQ-STO22.

\section{Resumo}

Objetivo: Validar a escala EORTC QLQ-STO22 para medir qualidade de vida em pacientes com cáncer de estômago na Colômbia. Métodos: Foi aplicado a 442 pacientes com diagnóstico de cáncer de estômago com idades entre 26 e 90 anos, o questionário EORTC QLQSTO22. Avaliou-se a validez de conteúdo com técnicas de análise fatorial; a validez de critério concorrente comparando pontuações das escalas QLQ-STO22 e FACIT-Ga; a consistência interna mediante alfa de Cronbach, a confiabilidade prova-reprova através do coeficiente de correlação de concordância de Lin e a sensibilidade à mudança após uma intervenção terapêutica comparando médias em medições repetidas. Resultados: a análise fatorial confirmou uma estrutura de cinco dimensões e a análise confirmatório mostrou aceitável ajuste do modelo. As correlações mais fortes entre a escala EORTC QLQ-STO22 e FACIT-Ga observaram-se com os domínios "Estado físico geral de saúde" e a subescala específica para cáncer gástrico da escala FACIT-Ga. Encontrou-se alta consistência interna com valores de alfa de Cronbach da escala global de 897. A medição da confiabilidade prova-reprova reportou valores do coeficiente de correlação de concordância de Lin entre .656 e .851. As diferenças entre pontuações antes e depois de uma intervenção terapêutica só foram significativas para os domínios "disfagia" e "restrições para comer”. Conclusão: a escala EORTC QLQ-STO22 é um instrumento válido e confiável para medir qualidade de vida em pacientes colombianos. Recomenda-se efetuar estudos adicionais para confirmar os resultados reportados durante a avaliação da sensibilidade à mudança desta escala em população colombiana.

Palavras-chave: qualidade de vida, câncer gástrico, validação de escalas, questionário, EORTC QLQ-STO22.

A pesar de que la incidencia global del cáncer gástrico ha disminuido en las últimas décadas, este continúa ocupando un lugar importante dentro de las causas de muerte relacionadas con cáncer alrededor del mundo. En el 2008 fue el cuarto cáncer más frecuente, con una estimación de cerca de un millón de nuevos casos de cáncer de estómago (Ferlay et al., 2013), con más del 70\% de los casos en países en desarrollo. En Colombia, para el 2012, según datos de la Organización Mundial de 
la Salud, el cáncer de estómago se constituyó en el segundo más frecuente en hombres, después del cáncer de próstata, y el cuarto en mujeres, después del de mama, cuello uterino y colon. Se estimó una tasa de incidencia de 18.9 por 100000 habitantes en hombres (3688 nuevos casos) y 9 por 100000 habitantes en mujeres (2209 nuevos casos) y fue considerada la patología oncológica con mayor número de muertes registradas en ambos sexos en la población colombiana para el 2012 (4981 muertes) (Ferlay et al., 2013).

De acuerdo con el anuario estadístico del Instituto Nacional de Cancerología (INC, 2014), en esa institución en el 2011 se diagnosticaron 260 casos nuevos de cáncer gástrico en hombres $(10.2 \%$ del total de casos nuevos de cáncer diagnosticados para el sexo masculino), y 176 casos en mujeres (4.9\% del total de casos nuevos de cáncer diagnosticados para el sexo femenino), con 80 muertes en hombres y 45 muertes en mujeres, registradas por esta misma causa (INC, 2014).

La resección quirúrgica, acompañada de adyuvancia con quimio o radioterapia continúa siendo la terapia primaria para todos los carcinomas gástricos (Abeloff, 2008), y desenlaces habituales como supervivencia global, libre de enfermedad, respuesta tumoral, entre otros, se mantienen como medidas significativas del efecto del tratamiento. Sin embargo, estos sistemas de evaluación tradicionales no miden los efectos que la enfermedad y su tratamiento ejercen en las dimensiones emocionales, espirituales y sociales del individuo con cáncer (Arraras et al., 2011). Para los pacientes con cáncer del tubo digestivo alto (esófago y estómago) estas consideraciones son especialmente importantes, porque la enfermedad y su tratamiento tienen una alta morbilidad e impacto en la calidad de vida (Blazeby \& Vickery, 2001; Sánchez, Alexander-Sierra \& Oliveros, 2012; Whistance \& Blazeby, 2011). Según algunos autores (Kaptein, Morita \& Sakamoto, 2005; Morita et al., 2006), aunque la supervivencia en pacientes con cáncer de estómago continúa siendo un desenlace crucial, la "cantidad" de supervivencia se ha ido complementando frecuentemente con datos sobre la "calidad" de la supervivencia. La calidad de vida reportada por los mismos pacientes se ha establecido como un resultado clínico de primer orden, tanto en la práctica médica diaria como dentro de estudios de investigación, y se ha considerado que aporta información útil en el pronóstico de pacientes con cáncer gástrico (Park et al., 2008).

Diversos instrumentos de medición para calidad de vida en pacientes oncológicos han sido y siguen siendo desarrollados por organizaciones internacionales como la Functional Assessment of Chronic Illness Therapy (FACIT) o la European Organization for Research and Treatment of Cancer (EORTC) (Aaronson et al., 1993; Cella et al., 1993). Estas organizaciones proveen instrumentos y aproximaciones similares para la evaluación de la calidad de vida relacionada con la salud en pacientes con cáncer. Ambos organismos han desarrollado módulos específicos para medir calidad de vida en cáncer gástrico: el FACIT-Ga y el EORTC QLQ-STO22.

El cuestionario QLQ-STO22, desarrollado de acuerdo con las guías del grupo de calidad de vida de la EORTC (Blazeby et al., 2004; Blazeby \& Vickery, 2001; Vickery et al., 2001), ha sido traducido a diversos idiomas y validado en diferentes países (Morita, Kaptein, Oba \& Sakamoto, 2008; Oñate-Ocaña et al., 2009; Sadighi, Montazeri, Sedighi, Mohagheghi \& Froutan, 2009). Este es un instrumento ampliamente utilizado para evaluar calidad de vida relacionada con la salud después de tratamientos, especialmente quirúrgicos (Fuchs et al., 2015; Lee, Chung, Kwon \& Yu, 2014; Park et al., 2014; Shen et al., 2013).

Hasta donde tenemos conocimiento, actualmente no existen instrumentos validados que evalúen la calidad de vida en pacientes con cáncer de estómago en Colombia - existe una aproximación psicométrica de la escala EORTC QLQ C30 en pacientes de Colombia, pero no incluye el módulo específico de estómago (Portillo \& Restrepo, 2004)—. Con el 
presente estudio se busca disponer de una versión validada de la escala EORTC QLQ-STO22, una de las más utilizadas en investigación clínica en este tipo de patología en el entorno internacional, para su uso en la población colombiana afligida por esta enfermedad.

\section{Materiales y métodos}

\section{Pacientes}

Utilizando un muestreo no probabilístico por conveniencia se seleccionaron entre febrero de 2009 y mayo de 2012 los pacientes de nacionalidad colombiana cuya lengua materna fuera español colombiano, con cáncer de estómago confirmado por histología en cualquier estadio y tratamiento, manejados en el INC en los servicios de consulta externa, hospitalización y urgencias de los departamentos de gastroenterología, cirugía, oncología, radioterapia y cuidados paliativos. Pacientes con imposibilidad para tramitar los formatos por afectación cognitiva, alteraciones del sensorio o incapacidad física que impidiera la comprensión o diligenciamiento del formulario no fueron considerados elegibles para participar en el estudio. Previa explicación de los objetivos del estudio, y una vez aceptaron participar, los pacientes diligenciaron el cuestionario con la asistencia de un auxiliar de investigación entrenado en la implementación del instrumento.

\section{Descripción del cuestionario}

El instrumento sometido a validación corresponde a la escala EORTC QLQ-STO22 en su versión adaptada al español colombiano (disponible en http://groups.eortc.be/qol). Las puntuaciones en cada uno de los dominios e ítems individuales fueron calculadas de acuerdo con las instrucciones entregadas por la EORTC en su manual para calificación de escalas (Blazeby et al., 2004).

El cuestionario QLQ-STO22 consta de 22 ítems, consistentes en cinco escalas multiítem hipotéticas relacionadas con disfagia, dolor, reflujo, restricciones para comer y ansiedad, y 4 ítems individuales relacionados con problemas con el sentido del gusto, imagen corporal, boca seca y pérdida del cabello (Blazeby et al., 2004). El instrumento tiene un sistema de puntuación simple, en el cual cada ítem o pregunta es evaluado en una dimensión ordinal de 1 a 4, con una escala de respuesta que varía de $1=$ en absoluto a $4=$ mucho. Llevando a cabo una transformación lineal de todos los puntajes, estos se convierten a una escala de medida de 1 a 100. Para las escalas de síntomas específicos, como es el caso del módulo QLQ-STO22 y de ítems individuales, los instrumentos desarrollados por EORTC están diseñados de forma tal que una alta puntuación equivale a más o peores síntomas, es decir, a una peor calidad de vida o a un mayor problema.

\section{Análisis estadístico}

Para examinar la estructura de la escala QLQSTO22 se llevó a cabo un análisis factorial exploratorio sobre los 17 ítems comprendidos en los 5 dominios hipotéticos de la escala en estudio. Se dejaron por fuera de esta fase los ítems que conceptualmente no fueron incluidos dentro de ninguno de estos dominios y fueron propuestos como ítems individuales por los autores de la escala QLQSTO22. Se computó la matriz de correlaciones entre los ítems y se verificó la factorizabilidad de la matriz por medio del test de esfericidad de Bartlett y la prueba Kaiser-Meyer-Olkin.

Para mejorar la interpretabilidad de los factores se recurrió a técnicas de rotación oblicua tipo Promax. Para completar la evaluación de la validez de constructo de la escala QLQ-STO22 fue efectuado un análisis factorial confirmatorio mediante modelos de ecuaciones estructurales. Dada la naturaleza ordinal de las puntuaciones de los ítems, se llevaron a cabo métodos de estimación utilizando correlación policórica y matrices de covarianza asintótica. El ajuste del modelo hipotético y de modelos resultantes del análisis 
exploratorio se evaluó mediante las siguientes pruebas y puntos de corte: la razón $\mathrm{X}^{2} / g l<3$; Root Mean Square Error of Approximation (RMSEA) $<$.05; Standardized Root Mean Square Residual $($ SRMR $)<.05$; Non-Normed Fit Index (NNFI); Comparative Fit Index (CFI) con valores cercanos a 1 , y Critical N $(\mathrm{CN})>200$ (Diamantopoulos \& Siguaw, 2000). Para determinar la validez de criterio concurrente se aplicaron simultáneamente las escalas EORTC QLQ-STO22 y FACIT-Ga y se evaluó estadísticamente por medio el coeficiente de correlación de Spearman, al no cumplirse los supuestos de normalidad en la distribución de los datos.

La consistencia interna global de la escala QLQSTO22 y de cada uno los factores se midió con el coeficiente alfa de Cronbach. Se consideró un grado de moderado a alto de homogeneidad de .7 a .9, para garantizar aceptable consistencia interna e ítems no redundantes (Keszei, Novak \& Streiner, 2010; Streiner \& Norman, 2008). El cambio en el coeficiente alfa de Cronbach, al retirar cada ítem, también fue evaluado. Luego de 2 a 10 días de la primera medición y antes de ser sometidos los pacientes a cualquier intervención, se efectuó una segunda aplicación de la escala para evaluar la confiabilidad prueba-reprueba: esto se realizó por medio del coeficiente de concordancia de Lin (Lin y Torbeck, 1998; Lin, 1989).

Para establecer si el instrumento permite detectar variaciones en la calidad de vida de los pacientes después de ser sometidos a una intervención terapéutica o paliativa, se determinaron los cambios en las respuestas de los ítems luego del cambio en la condición clínica posterior a una intervención de este tipo. El método estadístico utilizado fue la prueba de rangos con signo de Wilcoxon, a fin de comparar la diferencia de las medianas de los puntajes en cada factor pre y postratamiento. Los análisis estadísticos se efectuaron con los programas Lisrel $8.8 \AA$ y Stata $12 \AA$.

El estudio para validación de esta escala fue aprobado y vigilado por el Comité de Ética del INC.

\section{Resultados}

Un total de 442 pacientes diagnosticados histológicamente con cáncer de estómago, y que cumplían con los demás criterios de elegibilidad, fueron incluidos en el estudio: 251 (57\%) hombres y 191 (43\%) mujeres; con una media de edad de 62.15 años (rango 28-89) y 60.01 años (rango 26-90), respectivamente. La tabla 1 describe las características demográficas de los participantes según el sexo.

Tabla 1

Características demográficas de los participantes según sexo

\begin{tabular}{|c|c|c|c|c|c|}
\hline \multicolumn{2}{|c|}{ Variable } & \multicolumn{2}{|c|}{$\begin{array}{l}\text { Hombres } \\
(N=251)\end{array}$} & \multicolumn{2}{|c|}{$\begin{array}{l}\text { Mujeres } \\
(N=191)\end{array}$} \\
\hline \multicolumn{2}{|c|}{ Edad (media $\pm D E)$} & \multicolumn{2}{|c|}{$62.15 \pm 11.08$} & \multicolumn{2}{|c|}{$60.01 \pm 13.39$} \\
\hline \multirow{4}{*}{$\begin{array}{l}\text { Ocupación } \\
(N[\%])\end{array}$} & Empleado & 40 & $(15.90)$ & 21 & $(11.00)$ \\
\hline & Cesante & 64 & $(25.50)$ & 26 & $(13.60)$ \\
\hline & Jubilado & 47 & $(18.70)$ & 22 & $(11.50)$ \\
\hline & Otro & 100 & $(39.80)$ & 122 & $(63.90)$ \\
\hline \multirow{3}{*}{$\begin{array}{l}\text { Afiliación } \\
(N[\%])\end{array}$} & Contributivo & 108 & $(43.00)$ & 88 & $(46.10)$ \\
\hline & Subsidiado & 124 & $(49.40)$ & 86 & $(45.00)$ \\
\hline & Otros & 19 & $(7.60)$ & 17 & $(8.90)$ \\
\hline \multirow{5}{*}{$\begin{array}{l}\text { Escolaridad } \\
(N[\%])\end{array}$} & Ninguna & 18 & $(7.20)$ & 12 & $(6.30)$ \\
\hline & Primaria & 160 & $(63.70)$ & 116 & $(60.70)$ \\
\hline & Secundaria & 51 & $(20.30)$ & 36 & (18.80) \\
\hline & Superior & 21 & $(8.40)$ & 27 & $(14.10)$ \\
\hline & Perdidos & 1 & $(.40)$ & 0 & $(.00)$ \\
\hline \multirow{2}{*}{$\begin{array}{l}\text { Tipo de } \\
\text { paciente } \\
(N[\%])\end{array}$} & Ambulatorio & 222 & $(88.40)$ & 172 & $(90.10)$ \\
\hline & Hospitalizado & 29 & (11.60) & 19 & $(9.90)$ \\
\hline \multirow{2}{*}{$\begin{array}{l}\text { Procedencia } \\
(N[\%])\end{array}$} & Bogotá & 112 & $(44.60)$ & 88 & $(46.10)$ \\
\hline & $\begin{array}{l}\text { Otros } \\
\text { municipios }\end{array}$ & 139 & $(55.40)$ & 103 & $(53.90)$ \\
\hline \multirow{2}{*}{$\begin{array}{l}\text { Cirugía } \\
(N[\%])\end{array}$} & Sí & 155 & $(61.80)$ & 124 & $(64.90)$ \\
\hline & No & 96 & $(38.20)$ & 67 & (35.10) \\
\hline \multirow{2}{*}{$\begin{array}{l}\text { Quimiotera- } \\
\text { pia }(N[\%])\end{array}$} & Sí & 84 & $(33.50)$ & 35 & $(18.30)$ \\
\hline & No & 167 & $(66.50)$ & 156 & $(81.70)$ \\
\hline \multirow{2}{*}{$\begin{array}{l}\text { Radiotera- } \\
\text { pia }(N[\%])\end{array}$} & Sí & 50 & (19.90) & 26 & $(13.60)$ \\
\hline & No & 201 & $(80.10)$ & 165 & (86.40) \\
\hline
\end{tabular}




\begin{tabular}{cccccc}
\hline \multirow{2}{*}{ Variable } & \multicolumn{2}{c}{$\begin{array}{c}\text { Hombres } \\
(N=251)\end{array}$} & \multicolumn{2}{c}{$\begin{array}{c}\text { Mujeres } \\
(N=191)\end{array}$} \\
\hline Edad (media $\pm D E)$ & \multicolumn{2}{c}{$62.15 \pm 11.08$} & $60.01 \pm 13.39$ \\
\hline & 0 & 2 & $(.80)$ & 3 & $(1.60)$ \\
& I & 26 & $(10.40)$ & 34 & $(17.80)$ \\
Estadio & II & 41 & $(16.30)$ & 20 & $(10.50)$ \\
& III $])$ & 39 & $(15.50)$ & 22 & $(11.50)$ \\
& IV & 25 & $(10.00)$ & 19 & $(9.90)$ \\
& Perdidos & 118 & $(47.00)$ & 93 & $(48.70)$ \\
\hline
\end{tabular}

\section{Validez de constructo}

De los 442 pacientes a quienes les fue aplicado el cuestionario QLQ-STO22, 5 fueron excluidos por presentar datos perdidos y el análisis se llevó a cabo sobre 437 observaciones. La aplicación del instrumento tuvo una mediana de 22 minutos (rango entre 9 y $33 \mathrm{~min}$ ). Se incluyeron en el análisis los 17 ítems que conforman las escalas o dominios hipotéticos y se computó la matriz de correlaciones entre los ítems. De acuerdo con los resultados de la prueba de esfericidad de Bartlett y de la prueba Kaiser-Meyer-Olkin $(\mathrm{KMO}=.907)$, se verificó la factorizabilidad de la matriz de correlaciones. Se efectuó un análisis factorial exploratorio mediante el método de extracción de factores principales, teniendo en cuenta un criterio de cambio de inercia se seleccionaron 5 factores, los cuales dieron cuenta del $68.4 \%$ de la varianza explicada. Adicionalmente, se practicó una rotación oblicua (Promax). Todos los ítems cargaron en el factor hipotético original, excepto por el ítem 40 “ ¿Ha tenido dificultad al eructar?", que tuvo una mejor carga factorial en el dominio de restricciones para comer. Casi todos los ítems presentaron cargas significativas $>.3$ en los cinco factores, a excepción de los ítems 32, 43 y 48, que tuvieron una carga factorial $<.3$ en el factor relacionado con reflujo. Las correlaciones entre los factores tuvieron valores entre .348 (disfagia y reflujo) y .692 (dolor, restricciones para comer). La tabla 2 muestra la solución factorial rotada.
Tabla 2

Matriz de factores rotados

\begin{tabular}{|c|c|c|c|c|c|}
\hline \multirow{2}{*}{ Ítem } & \multicolumn{5}{|c|}{ Factor } \\
\hline & 1 & 2 & 3 & 4 & 5 \\
\hline $\begin{array}{l}\text { sto } 31 \text { ¿Ha tenido algún } \\
\text { problema al comer alimentos } \\
\text { sólidos? }\end{array}$ & .510 & .667 & .599 & .533 & .312 \\
\hline $\begin{array}{l}\text { sto } 32 \text { ¿Ha tenido algún } \\
\text { problema al comer alimentos } \\
\text { licuados o blandos? }\end{array}$ & .421 & .928 & .578 & .413 & .276 \\
\hline $\begin{array}{l}\text { sto } 33 \text { ¿Ha tenido algún pro- } \\
\text { blema al beber líquidos? }\end{array}$ & .397 & .731 & .447 & .330 & .306 \\
\hline $\begin{array}{l}\text { sto } 34 \text { ¿Ha sentido molestias } \\
\text { mientras comía? }\end{array}$ & .684 & .668 & .651 & .443 & .354 \\
\hline $\begin{array}{l}\text { sto } 35 \text { ¿Ha sentido dolor en } \\
\text { la zona del estómago? }\end{array}$ & .939 & .459 & .583 & .557 & .424 \\
\hline $\begin{array}{l}\text { sto } 36 \text { ¿Ha sentido molestias } \\
\text { en la zona del estómago? }\end{array}$ & .907 & .513 & .617 & .539 & .473 \\
\hline $\begin{array}{l}\text { sto } 37 \text { ¿Ha tenido sensación } \\
\text { de hinchazón en el abdomen? }\end{array}$ & .652 & .313 & .520 & .412 & .478 \\
\hline $\begin{array}{l}\text { sto } 38 \text { ¿Ha tenido problemas } \\
\text { de subida de acidez o bilis (sa- } \\
\text { bor amargo) a su boca? }\end{array}$ & .417 & .345 & .473 & .402 & .840 \\
\hline $\begin{array}{l}\text { sto } 39 \text { ¿Ha tenido indiges- } \\
\text { tión con acidez o ardor de } \\
\text { estómago? }\end{array}$ & .535 & .353 & .495 & .414 & .861 \\
\hline $\begin{array}{l}\text { sto } 40 \text { ¿Ha tenido dificultad } \\
\text { al eructar? }\end{array}$ & .403 & .375 & .487 & .328 & .347 \\
\hline $\begin{array}{l}\text { sto } 41 \text { ¿Se ha sentido saciado } \\
\text { al poco tiempo de comenzar } \\
\text { a comer? }\end{array}$ & .427 & .356 & .653 & .416 & .357 \\
\hline $\begin{array}{l}\text { sto } 42 \text { ¿Ha tenido dificultad } \\
\text { al disfrutar de sus comidas? }\end{array}$ & .630 & .555 & .810 & .570 & .410 \\
\hline $\begin{array}{l}\text { sto } 43 \text { ¿Tardó mucho tiempo } \\
\text { en acabar de comer? }\end{array}$ & .361 & .423 & .618 & .423 & .297 \\
\hline $\begin{array}{l}\text { sto } 46 \text { ¿Ha tenido dificultad } \\
\text { al comer delante de otras } \\
\text { personas? }\end{array}$ & .387 & .426 & .539 & .374 & .293 \\
\hline $\begin{array}{l}\text { sto } 47 \text { ¿Ha pensado en su } \\
\text { enfermedad? }\end{array}$ & .476 & .374 & .541 & .734 & .376 \\
\hline $\begin{array}{l}\text { sto } 48 \text { ¿Se ha preocupado } \\
\text { por tener un peso demasiado } \\
\text { bajo? }\end{array}$ & .417 & .433 & .478 & .549 & .239 \\
\hline $\begin{array}{l}\text { sto } 50 \text { ¿Ha estado preocupa- } \\
\text { do por su salud futura? }\end{array}$ & .480 & .373 & .514 & .824 & .367 \\
\hline
\end{tabular}


Se efectuó un análisis factorial confirmatorio para evaluar dos modelos diferentes. El modelo 1 correspondió a la estructura original de la escala QLQ-STO22; el modelo 2, a la estructura sugerida por el análisis factorial exploratorio. El método de estimación utilizado fue máxima verosimilitud. Las variables latentes fueron estandarizadas, con varianza fijada en 1 . Las figuras 1 y 2 muestran las características de los diagramas de trayectoria de cada uno de los modelos.

La tabla 3 presenta los valores de los índices de ajuste de cada uno de los dos modelos analizados. Para ambos casos hubo valores de $X^{2} /$ $g l<3$. En ninguno de los casos los índices RMSEA y SRMR fueron < .05; adicionalmente, se encontraron valores de RMSEA por encima de

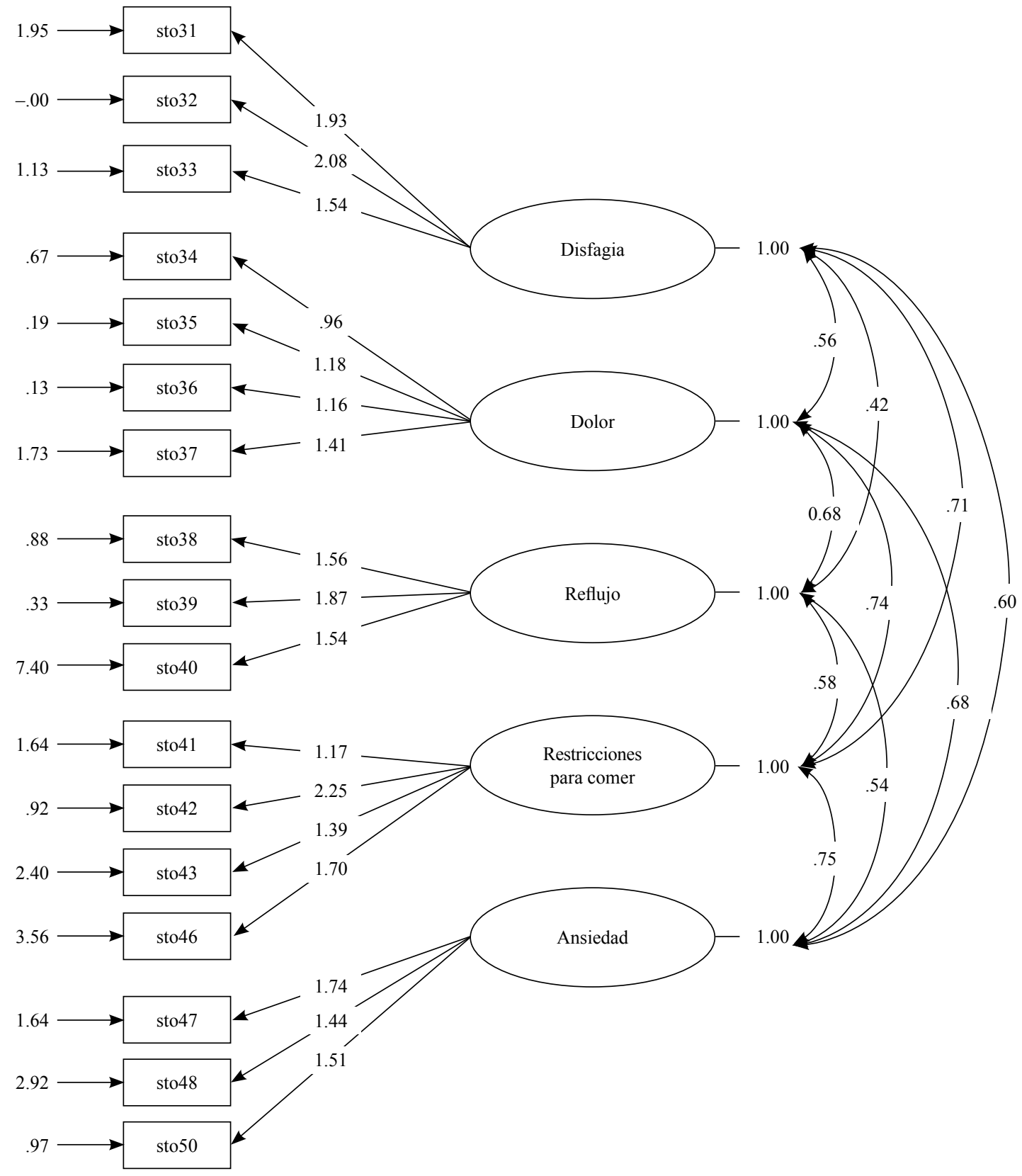

Figura 1. Diagrama de trayectoria modelo 1: modelo original 


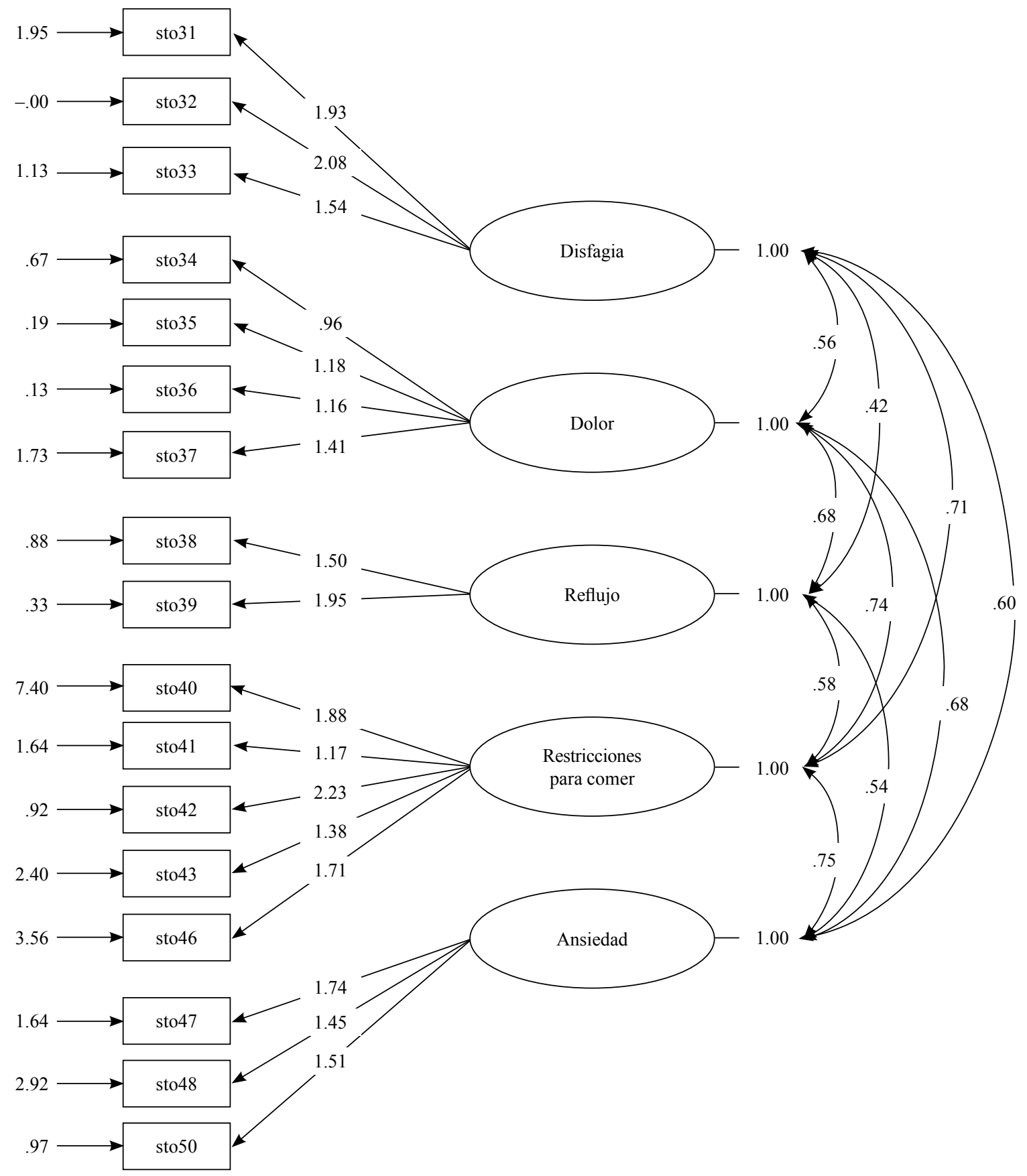

Figura 2. Diagrama de trayectoria modelo 2: modelo ajustado por el análisis exploratorio

Tabla 3

Estadisticos de ajuste correspondientes al análisis factorial confirmatorio

\begin{tabular}{lccccccccc}
\hline & $X^{2}$ & $g l$ & $X^{2} / g l$ & RMSEA & NNFI & CFI & SRMR & ECVI & CN \\
\hline Modelo 1 & 229.56 & 109 & 2.106 & .113 & .989 & .991 & .088 & .718 & 281.975 \\
Modelo 2 & 205.74 & 109 & 1.888 & .106 & .991 & .993 & .067 & .665 & 314.501 \\
\hline
\end{tabular}


.10 , lo que sugiere un pobre ajuste. El modelo 2 obtuvo los índices SRMR y ECVI más bajos, con el mayor valor de CN. Los índices NNFI y CFI se situaron por encima de .9 en los dos modelos analizados, siendo mayores estos valores en el modelo 2 .

\section{Validez de criterio concurrente}

Las escalas EORTC QLQ-STO22 y FACIT-Ga se administraron de forma paralela a un total de 250 pacientes. Las correlaciones más fuertes se observaron con los dominios "Estado físico general de salud" y "Otras preocupaciones" (subescala cáncer gástrico) de la escala FACIT-Ga. El mejor coeficiente de correlación se encontró entre "Dolor" y "Estado físico general de salud" ( $r=-.739$; $p<.001)$ (tabla 4).

\section{Consistencia interna}

La consistencia global de escala fue .897. Los valores del coeficiente alfa, al ser retirado cada ítem, variaron entre .87 y .901 (tabla 5). El ítem 44 “Ha tenido la boca seca?”, al ser retirado, incrementó el nivel de consistencia global a un valor ligeramente mayor a 0.9. Los valores del coeficiente alfa de Cronbach obtenido para cada uno de los factores fue: disfagia: .790; dolor: .866; reflujo: .723; restricciones para comer: .746, y ansiedad: .741.

Tabla 4

Valores de correlación entre las escalas QLQ-STO22 y FACIT-Ga

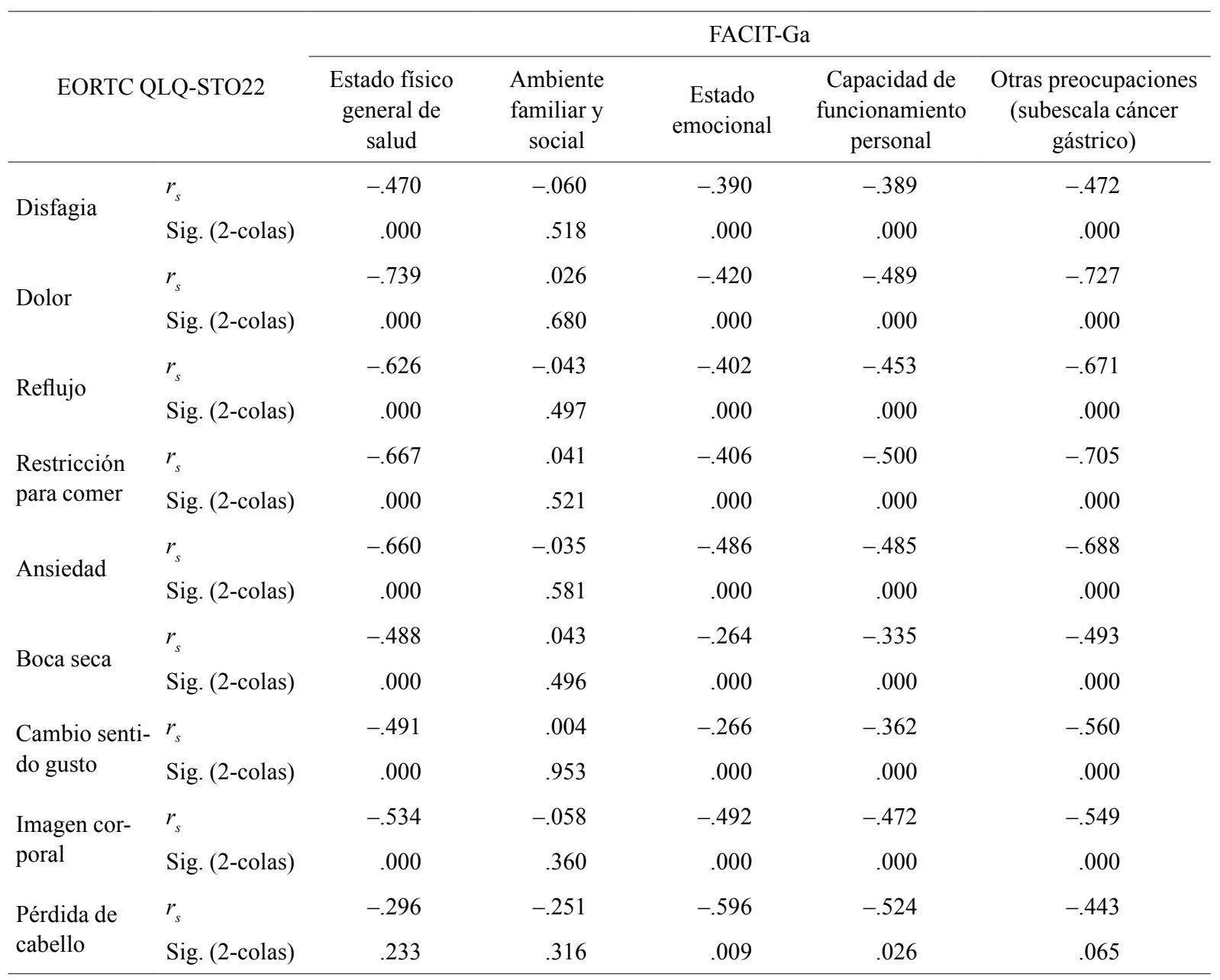


Tabla 5

Valores de media de la escala y de alfa de Cronbach al retirar items

\begin{tabular}{ccc}
\hline Ítem & $\begin{array}{c}\text { Media de la escala si } \\
\text { se retira el ítem }\end{array}$ & $\begin{array}{c}\text { Alfa de Cronbach si se } \\
\text { retira el ítem }\end{array}$ \\
\hline sto31 & 43.52 & .890 \\
sto32 & 44.29 & .891 \\
sto33 & 44.25 & .892 \\
sto34 & 43.79 & .890 \\
sto35 & 43.43 & .888 \\
sto36 & 43.46 & .889 \\
sto37 & 43.68 & .891 \\
sto38 & 43.63 & .893 \\
sto39 & 43.64 & .892 \\
sto40 & 44.00 & .895 \\
sto41 & 43.52 & .892 \\
sto42 & 43.68 & .887 \\
sto43 & 43.58 & .894 \\
sto44 & 43.41 & .901 \\
sto45 & 43.98 & .892 \\
sto46 & 44.38 & .894 \\
sto47 & 43.03 & .891 \\
sto48 & 43.35 & .893 \\
sto49 & 43.44 & .893 \\
sto50 & 43.03 & .890 \\
\hline
\end{tabular}

\section{Confiabilidad prueba-reprueba}

A un grupo de 75 pacientes se les administró el cuestionario en dos momentos consecutivos, en un intervalo de tiempo entre 2 y 10 días, con una diferencia media de tiempo entre las dos mediciones de 5.95 días ( $D E 1.18)$. El ítem 52, relacionado con pérdida del cabello, no fue incluido en este análisis, pues la respuesta a este ítem está condicionada a una respuesta positiva en el ítem 51 “ ¿Se le cayó algo de pelo?". La tabla 6 muestra los resultados de la medición de la confiabilidad prueba-reprueba para cada una de las subescalas y de los ítems individuales.
Tabla 6

Coeficientes de correlación concordancia de Lin

\begin{tabular}{lccc}
\hline \multicolumn{1}{c}{$\begin{array}{c}\text { Subescala/item } \\
\text { individual }\end{array}$} & $\rho_{c}{ }^{a}$ & \multicolumn{2}{c}{ IC 95\% } \\
\hline Disfagia & .851 & .789 & .913 \\
Dolor & .756 & .658 & .854 \\
Reflujo & .656 & .529 & .782 \\
Restricciones para & .774 & .683 & .865 \\
comer & .825 & .751 & .898 \\
Ansiedad & .732 & .624 & .839 \\
Boca seca & .719 & .608 & .831 \\
Cambio sentido gusto & .715 & .603 & .827 \\
Imagen corporal & &
\end{tabular}

\section{Sensibilidad al cambio}

Esta evaluación se llevó a cabo a partir de datos de 45 pacientes a quienes se les aplicó el cuestionario en dos momentos diferentes de medición pre y postratamiento. El $55.6 \%$ de los pacientes $(N=25)$ fueron hombres. La media de edad en este grupo fue de 64.1 años ( $D E=12.5$ años). La mayoría de los pacientes se encontraban sin ninguna vinculación laboral $(68.9 \% ; N=31)$. En cuanto a su afiliación al sistema de salud, se encontró que 23 (51.1\%) estaban en régimen contributivo; 21 (46.7\%), en subsidiado, y uno (2.2\%), en otros regímenes. Solo 4 pacientes estaban hospitalizados $(8.9 \%)$.

La distribución según estadio clínico en este grupo fue: estadio 0: $2.2 \%(N=1)$; estadio I: $33.3 \%$ $(N=15)$; estadio II: $20 \%(N=9)$, y estadios III y IV: $22.2 \%$, respectivamente $(N=10$ en cada estadio). De los 45 pacientes, 31 (68.9\%) habían sido sometidos a cirugía gástrica, 11 (24.4\%) iniciaron esquemas de quimioterapia, $2(4.4 \%)$ recibieron tratamiento con radioterapia y $1(2.2 \%)$ fue sometido a cuidados paliativos. La diferencia media de tiempo entre las mediciones pre y postratamiento fue de 141.44 días ( $D E$ 66.14); la media del tiempo desde la intervención o tratamiento hasta la segunda medición fue de 74.1 días ( $D E$ 45.1). La media de 
las puntuaciones pre y postratamiento para cada dominio e ítem individual son presentados en la tabla 7. Las diferencias entre las puntuaciones antes y después de una intervención terapéutica solo fueron significativas para los dominios de disfagia y restricciones para comer.

\section{Discusión}

En la actualidad, es ampliamente reconocido que la medición de la calidad de vida en pacientes con enfermedades crónicas, especialmente en el caso de las enfermedades oncológicas, ha ganado importancia y se ha transformado en un pilar de apoyo para la toma de decisiones clínicas. El considerar la calidad de vida un desenlace prioritario en cáncer, y su progresiva incorporación dentro de estudios de investigación clínica mundial, han hecho necesario contar con instrumentos validados que permitan evaluar este constructo en los pacien- tes con cáncer desde su propia subjetividad. Así, el propósito de este estudio fue estudiar las propiedades psicométricas de la versión adaptada al español colombiano de la escala EORTC QLQ-STO22 para medir calidad de vida en una muestra de pacientes colombianos con cáncer gástrico.

Para esto se completaron las diferentes etapas del proceso de validación de escalas. Para una estructura de cinco factores, se revela una adecuada representación de los ítems por los dominios teóricos originales, aun cuando el ítem 40, ¿ ¿Ha tenido dificultad al eructar?", tuvo una mayor carga factorial en el dominio de restricciones para comer. Este agrupamiento de factores, aunque no se corresponde fielmente con la estructura original, resulta teóricamente plausible. No solo desde las diferencias culturales bien conocidas que existen frente a la liberación o expulsión de gases estomacales, como se define el eructo, sino también desde el punto de vista de la percepción de esta expulsión

Tabla 7

Media de las puntuaciones antes y después de tratamiento

\begin{tabular}{|c|c|c|c|c|c|c|c|c|c|}
\hline \multirow{2}{*}{\multicolumn{3}{|c|}{ Dominio/ítem individual }} & \multicolumn{4}{|c|}{ Pretratamiento } & \multicolumn{3}{|c|}{ Postratamiento } \\
\hline & & & \multicolumn{2}{|r|}{ Media } & \multicolumn{2}{|l|}{$D E$} & \multicolumn{2}{|l|}{ Media } & $D E$ \\
\hline \multicolumn{3}{|l|}{ Disfagia } & \multicolumn{2}{|r|}{20.00} & \multicolumn{2}{|l|}{25.46} & \multicolumn{2}{|l|}{31.85} & 29.35 \\
\hline \multicolumn{3}{|l|}{ Dolor } & \multicolumn{2}{|r|}{34.44} & \multicolumn{2}{|l|}{28.90} & \multicolumn{2}{|l|}{32.77} & 29.69 \\
\hline \multicolumn{3}{|l|}{ Reflujo } & \multicolumn{2}{|r|}{24.69} & \multicolumn{2}{|l|}{24.14} & \multicolumn{2}{|l|}{29.38} & 28.44 \\
\hline \multicolumn{3}{|c|}{ Restricciones para comer } & \multicolumn{2}{|r|}{26.60} & \multicolumn{2}{|l|}{25.81} & \multicolumn{2}{|l|}{36.66} & 28.39 \\
\hline \multicolumn{3}{|l|}{ Ansiedad } & \multicolumn{2}{|r|}{48.64} & \multicolumn{2}{|l|}{29.80} & \multicolumn{2}{|l|}{51.60} & 25.53 \\
\hline \multicolumn{3}{|l|}{ Boca seca } & \multicolumn{2}{|r|}{33.33} & \multicolumn{2}{|l|}{35.53} & \multicolumn{2}{|l|}{34.81} & 36.20 \\
\hline \multicolumn{3}{|c|}{ Cambio en sentido del gusto } & \multicolumn{2}{|r|}{20.00} & \multicolumn{2}{|l|}{32.87} & \multicolumn{2}{|l|}{28.14} & 35.50 \\
\hline \multicolumn{3}{|c|}{ Imagen corporal } & \multicolumn{2}{|r|}{27.40} & 34.29 & & 34.81 & & 36.20 \\
\hline Pérdida de cal & ello & & & 56.24 & 29.10 & & 42.22 & & 36.65 \\
\hline & & & & Test de $\mathrm{V}$ & ilcoxon & & & & \\
\hline & Disfagia & Dolor & Reflujo & $\begin{array}{l}\text { Restriccio- } \\
\text { nes para } \\
\text { comer }\end{array}$ & Ansiedad & Boca seca & $\begin{array}{l}\text { Cambios } \\
\text { sentido del } \\
\text { gusto }\end{array}$ & $\begin{array}{l}\text { Imagen } \\
\text { corporal }\end{array}$ & $\begin{array}{l}\text { Pérdida de } \\
\text { cabello }\end{array}$ \\
\hline$Z$ & -2.652 & -.287 & -1.149 & -2.576 & -1.096 & -.274 & -1.426 & -1.064 & -1.594 \\
\hline Sig. (2-colas) & .008 & .774 & .251 & .010 & .273 & .784 & .154 & .287 & .111 \\
\hline
\end{tabular}


de gases como un "hábito" o "acción" saludable frente a la distensión abdominal y la sensación de plenitud gástrica.

Los resultados del análisis factorial confirmatorio también defendieron la solución de una estructura multidimensional de la escala conformada por cinco factores y se encontró que el modelo que mejor se ajustó a los datos fue el modelo propuesto por el análisis exploratorio; no obstante, los valores que tomaron los índices de ajuste para los dos modelos analizados no pusieron de manifiesto un ajuste perfecto de ninguno de estos con los datos observados, aunque se alcanzó una adecuada representación de los datos y un ajuste relativamente razonable. Cabe anotar que los índices de ajuste para el modelo propuesto por el análisis exploratorio no se encontraron muy lejos de los valores que tomaron estos índices para el modelo hipotético, el cual también permaneció demostrando un ajuste razonable a los datos observados.

De los estudios de validación de la escala QLQSTO22 disponibles hasta la fecha de realización de este trabajo, solo en el estudio de validación japonés, conducido por Morita (Morita et al., 2008), fue llevado a cabo un análisis factorial exploratorio para evaluar la estructura del instrumento. La estructura sometida a evaluación correspondió a la versión preliminar y no a la modificada por Blazeby posterior a la determinación de sus propiedades psicométricas, la cual se constituyó en la versión final de la escala QLQL-STO22.

La validez de criterio concurrente exhibió correlaciones apropiadas entre distintos dominios de las escalas QLQ-STO22 y FACIT-Ga, al presentar correlaciones de moderadas a fuertes entre todos los factores e ítems individuales del cuestionario QLQL-STO22 y la subescala específica para cáncer gástrico del cuestionario FACIT-Ga. Solo el dominio de dolor obtuvo su mayor correlación con un dominio diferente a esta subescala de cáncer gástrico. Así, el factor de estado físico general, que incluye una pregunta explícita sobre dolor entre otras relacionadas con temas como falta de energía, náuseas o molestias por efectos secundarios del tratamiento, se constituyó en el factor con el cual el dominio de dolor logró su más alta correlación.

El dominio concerniente a ansiedad presentó una correlación moderada con el factor de estado emocional de la escala FACIT-Ga, el cual comprende 6 ítems o preguntas que intentan medir con mayor especificidad la condición emocional del paciente, pero mostró una correlación fuerte con la subescala de cáncer gástrico, que contiene ítems como "estoy bajando de peso" o "debido a mi enfermedad, me resulta difícil hacer planes para el futuro", cuya redacción o formulación se advierte en consonancia con la de los ítems del dominio de ansiedad de la escala en estudio: “ ¿Se ha preocupado por tener un peso demasiado bajo?” o “ ¿Ha estado preocupado por su salud futura?".

La consistencia interna global de la escala fue adecuada, con un valor del alfa de Cronbach de .897, que significa homogeneidad entre los ítems sin llegar a ser redundantes. Los resultados arrojados indican que todos los ítems contribuyen a un alto valor de confiabilidad, sin que se evidencie una reducción sustancial en el coeficiente alfa, al ser retirados. Solo el retiro del ítem "¿Ha tenido la boca seca?" aumentó ligeramente el valor del coeficiente, pero no de manera importante. Para cada uno de los dominios se obtuvieron, asimismo, valores apropiados del coeficiente alfa. El dominio que mostró una mayor consistencia interna fue el relacionado con dolor; el de menor consistencia fue el relacionado con reflujo. Los hallazgos en esta etapa del proceso de validación son acordes con los reportados por otros autores (Oñate-Ocaña et al., 2009). Al realizar la validación de la misma escala en población mexicana, Oñate-Ocaña et al. (2009) reportaron valores de alfa de Cronbach mayores a 7 para todos los dominios, excepto para el relacionado con reflujo en el que obtuvo un alfa menor a .6, y el dominio que mostró mayor consistencia fue el relacionado con dolor, con un alfa por encima de .8 . 
Respecto a la confiabilidad de la prueba, los factores disfagia y ansiedad se manifestaron claramente consistentes a través de las mediciones repetidas. El resto de dominios e ítems individuales, excepto el dominio relacionado con reflujo, mostraron aceptables coeficientes de correlación en las medidas repetidas. Al evaluar la sensibilidad al cambio solo se encontraron diferencias significativas en los dominios de disfagia y restricciones para comer. En general, las puntuaciones de las mediciones postratamiento revelaron una percepción de empeoramiento de los síntomas relacionados, no únicamente con disfagia y restricciones para comer, sino también en los dominios de reflujo y ansiedad, y en todos los ítems individuales, a excepción del asociado con pérdida de cabello. En otras poblaciones, no obstante, se ha reportado una adecuada sensibilidad al cambio, con diferencias estadísticamente significativas, al comparar puntajes obtenidos antes y después de una intervención entre grupos de pacientes sometidos a diferentes tipos de tratamiento, en la mayoría de los dominios e ítems individuales $(10-13,20,21)$.

En la mayor parte de estas poblaciones se llevaron a cabo varias mediciones de la calidad de vida, generalmente a los meses $1,3,6,9$ y 12 postratamiento, lo que evidencia cambios significativos en las puntuaciones de las escalas funcionales y de síntomas (Kim et al., 2012; Kong, Kwon \& Yu, 2012; Lee, Hur \& Kim, 2010). La pobre capacidad para detectar el cambio puede corresponder a una limitación de este estudio, ya que solo se practicaron dos medidas repetidas y no se estandarizaron las intervenciones ni los tiempos de evaluación posterapia y, eventualmente, a un insuficiente tamaño de muestra para detectar las pequeñas diferencias encontradas.

En conclusión, el proceso de validación de la escala QLQ-STO22 para su uso en Colombia muestra que el instrumento tiene una validez aceptable y una confiabilidad adecuada, lo cual revela una estructura ligeramente diferente a la propuesta y validada por los autores (Blazeby et al., 2004). Las propiedades psicométricas encontradas, así como el tiempo necesario para su diligenciamiento y el no requerimiento de condiciones de escolaridad particulares, hacen de este instrumento una herramienta aplicable en escenarios de investigación clínica y de atención de pacientes. Se proponen estudios adicionales que evalúen de manera más precisa la sensibilidad al cambio del instrumento y que utilicen otras estrategias de análisis basadas en teoría de respuesta al ítem.

\section{Referencias}

Aaronson, N. K., Ahmedzai, S., Bergman, B., Bullinger, M., Cull, A., Duez, N. J., et al. (1993). The European Organization for Research and Treatment of Cancer QLQ-C30: A quality-of-life instrument for use in international clinical trials in oncology. Journal of the National Cancer Institute, 85(5), 365-376.

Abeloff, M. D. (2008). Abeloff's clinical oncology (4th ed.). Philadelphia: Churchill Livingstone/ Elsevier.

Arraras, J. I., Arias de la Vega, F., Illarramendi, J. J., Manterola, A., Salgado, E., Dominguez, M. A., \& Vera, R. (2011). [Health-related quality of life in the oncology departments of the hospital of Navarra. The EORTC Quality of Life Group]. Anales del Sistema Sanitario de Navarra, 34(1), 9-20.

Blazeby, J. M., Conroy, T., Bottomley, A., Vickery, C., Arraras, J., Sezer, O., Quality of Life, G. (2004). Clinical and psychometric validation of a questionnaire module, the EORTC QLQ-STO 22, to assess quality of life in patients with gastric cancer. European Journal of Cancer, 40(15), 2260-2268. doi: 10.1016/j. ejca.2004.05.023

Blazeby, J. M., \& Vickery, C. W. (2001). Quality of life in patients with cancers of the upper gastrointestinal tract. Expert Review of Anticancer Therapy, 1(2), 269-276. doi: 10.1586/14737140.1.2.269 
Cella, D. F., Tulsky, D. S., Gray, G., Sarafian, B., Linn, E., Bonomi, A., et al. (1993). The Functional Assessment of Cancer Therapy scale: Development and validation of the general measure. Journal of Clinical Oncology: Official Journal of the American Society of Clinical Oncology, 11(3), 570-579.

Diamantopoulos, A., \& Siguaw, J. A. (2000). Introducing Lisrel: A guide for the uninitiated. London: Sage.

Ferlay, J., Soerjomataram, I., Ervik, M., Dikshit, R., Eser, S., Mathers, C., Bray, F. (2013). Cancer incidence and mortality worldwide: IARC CancerBase No. 11. GLOBOCAN 2012 v 1.0. Recuperado de http://globocan.iarc.fr

Fuchs, H., Holscher, A. H., Leers, J., Bludau, M., Brinkmann, S., Schroder, W., Gutschow, C. A. (2015). Long-term quality of life after surgery for adenocarcinoma of the esophagogastric junction: Extended gastrectomy or transthoracic esophagectomy? Gastric Cancer: Official Journal of the International Gastric Cancer Association and the Japanese Gastric Cancer Association . doi: 10.1007/s10120-015-0466-3

Instituto Nacional de Cancerología (INC). (2014). Anuario estadístico 2011 (vol. 9). Bogotá: autor. Recuperado de http://www.cancer.gov.co

Kaptein, A. A., Morita, S., \& Sakamoto, J. (2005). Quality of life in gastric cancer. World Journal of Gastroenterology: WJG, 11(21), 3189-3196.

Keszei, A. P., Novak, M., \& Streiner, D. L. (2010). Introduction to health measurement scales. Journal of Psychosomatic Research, 68(4), 319323. doi: 10.1016/j.jpsychores.2010.01.006

Kim, A. R., Cho, J., Hsu, Y. J., Choi, M. G., Noh, J. H., Sohn, T. S., Kim, S. (2012). Changes of quality of life in gastric cancer patients after curative resection: a longitudinal cohort study in Korea. Annals of Surgery, 256(6), 1008-1013. doi: 10.1097/SLA.0b013e31827661c9

Kong, H., Kwon, O. K., \& Yu, W. (2012). Changes of quality of life after gastric cancer surgery.
Journal of gastric cancer, 12(3), 194-200. doi: 10.5230/jgc.2012.12.3.194

Lee, J., Hur, H., \& Kim, W. (2010). Improved longterm quality of life in patients with laparoscopy-assisted distal gastrectomy with jejunal pouch interposition for early gastric cancer. Annals of Surgical Oncology, 17(8), 2024-2030. doi: 10.1245/s10434-010-1095-z

Lee, S. S., Chung, H. Y., Kwon, O. K., \& Yu, W. (2014). Quality of life in cancer survivors 5 years or more after total gastrectomy: a case-control study. International Journal of Surgery, 12(7), 700-705. doi: 10.1016/j.ijsu.2014.05.067

Lin, L., \& Torbeck, L. D. (1998). Coefficient of accuracy and concordance correlation coefficient: new statistics for methods comparison. PDA Journal of Pharmaceutical Science and Technology/PDA, 52(2), 55-59.

Lin, L. I. (1989). A concordance correlation coefficient to evaluate reproducibility. Biometrics, 45(1), 255-268.

Morita, S., Kaptein, A. A., Oba, K., \& Sakamoto, J. (2008). The domain structure of the EORTC QLQ-STO22 supported by Japanese validation data. Psychooncology, 17(5), 474-479. doi: 10.1002/pon.1256

Morita, S., Kaptein, A. A., Tsuburaya, A., Kodera, Y., Matsui, T., \& Sakamoto, J. (2006). Assessment and data analysis of health-related quality of life in clinical trials for gastric cancer treatments. Gastric Cancer: Official Journal of the International Gastric Cancer Association and the Japanese Gastric Cancer Association, 9(4), 254-261. doi: 10.1007/s10120-006-0400-9

Oñate-Ocaña, L. F., Alcántara-Pilar, A., Vilar-Compte, D., García-Hubard, G., Rojas-Castillo, E., Alvarado-Aguilar, S., Aiello-Crocifoglio, V. (2009). Validation of the Mexican Spanish version of the EORTC C30 and STO22 questionnaires for the evaluation of health-related quality of life in patients with gastric cancer. 
Annals of Surgical Oncology, 16(1), 88-95. doi: 10.1245/s10434-008-0175-9

Park, J. Y., Eom, B. W., Jo, M. J., Yoon, H. M., Ryu, K. W., Kim, Y. W., Lee, J. H. (2014). Health-related quality of life after robot-assisted distal gastrectomy in early gastric cancer. World Journal of Surgery, 38(5), 1112-1120. doi: 10.1007/ s00268-013-2390-1

Park, S. H., Cho, M. S., Kim, Y. S., Hong, J., Nam, E., Park, J., Lee, W. K. (2008). Self-reported health-related quality of life predicts survival for patients with advanced gastric cancer treated with first-line chemotherapy. Quality of Life Research: An International Journal of Quality of Life Aspects of Treatment, Care and Rehabilitation, 17(2), 207-214. doi: 10.1007/ s11136-008-9307-8

Portillo, C., \& Restrepo, M. (2004). Evaluación del cuestionario de calidad de vida EORTC QLQ C30 dentro de un estudio con pacientes adultos de centros de oncología de Bogotá. Avances en Medición, 2(1), 51-61.

Sadighi, S., Montazeri, A., Sedighi, Z., Mohagheghi, M. A., \& Froutan, H. (2009). Quality of life in patients with gastric cancer: translation and psychometric evaluation of the Iranian version of EORTC QLQ-STO22. BMC Cancer, 9, 305. doi: 10.1186/1471-2407-9-305

Fecha de recepción: 12 de julio de 2014 Fecha de aceptación: 18 de abril de 2015
Sánchez, R., Alexander-Sierra, F., \& Oliveros, R. (2012). Relationship between quality of life and clinical status in patients with gastrointestinal cancer. Revista Española de Enfermedades Digestivas, 104(11), 584-591.

Shen, C., Yang, H., Zhang, B., Chen, H., Chen, Z., \& Chen, J. (2013). Changes of quality of life after gastric tube reconstruction in adenocarcinoma of the esophagogastric junction. Pakistan Journal of Medical Sciences, 29(5), 1193-1198.

Streiner, D. L., \& Norman, G. R. (2008). Health measurement scales: A practical guide to their development and use (4th ed.). Oxford: Oxford University Press.

Vickery, C. W., Blazeby, J. M., Conroy, T., Arraras, J., Sezer, O., Koller, M., Group, E. Q. o. L. (2001). Development of an EORTC disease-specific quality of life module for use in patients with gastric cancer. European Journal of Cancer, 37(8), 966-971.

Whistance, R. N., \& Blazeby, J. M. (2011). Systematic review: quality of life after treatment for upper gastrointestinal cancer. Current Opinion in Supportive and Palliative Care, 5(1), 37-46. doi: 10.1097/SPC.0b013e3283436ecb 